\title{
EFICIÊNCIA FINANCEIRA, ATORES E INTERAÇÕES: UM ESTUDO DO FLUXO DE JOGADORES ENTRE CLUBES E AS EQUIPES SEMIFINALISTAS DE SÃO PAULO EM 2017
}

\author{
Edimilson Eduardo da Silva ${ }^{1}$, Agnaldo Antonio dos Santos, Marco Antônio Pinheiro da Silveira, Paulo Jorge Reis Mourão \\ Universidade Municipal de São Caetano do Sul - USCS, São Caetano do Sul (Brasil)
}

Universidade do Minho, Braga, (Portugal)

\section{DETALHES DO ARTIGO}

\section{Histórico do Artigo:}

Recebido: 29 de março de 2019

Aceito: 23 de setembro de 2019

Disponível online: 01 de jan. de 2020

Sistema de revisão "Double blind review"

Editor Científico

Ilan Avrichir

\section{Palavras-chaves:}

Eficiência Financeira

Análise de Redes Sociais

Fluxo de Jogadores

\begin{abstract}
RESUMO
Objetivo: apresentar os índices de eficiência financeira dos clubes brasileiros, os atores e as interações que se destacaram nos fluxos de jogadores com os clubes semifinalistas de São Paulo em 2017.

Método: na primeira etapa, extraíram-se informações sobre fluxos de jogadores e, na segunda etapa, dados sobre a receita operacional, fornecedores, custo de atletas profissionalizados e atletas não profissionalizados nos clubes do Brasil. Principais resultados - os escores eficiência financeira forneceram evidências do nível máximo de uso de recursos de atletas profissionalizados e não profissinalizados na geração de receita operacional no Palmeiras e Atlético Mineiro. Em relação aos atores tem-se que os clubes Grêmio Osasco Audax e Cruzeiro Esporte Clube disponibilizaram mais jogadores para os clubes semifinalistas. Nos fluxos de jogadores entre clubes do Brasil e de outros países, destacaram-se as equipes da Itália e de Portugal.

Relevância/originalidade: demonstrar os scores de eficiência máximo dos clubes no uso de recursos na geração de receita operacional, os atores de destaque e as interações nos fluxos de jogadores.

Contribuições metodológicas:uso simultâneo de técnicas paramétricas e não paramétricas como a Regressão Linear Múltipla, a Análise Envoltória de Dados (DEA) e a Análise de Redes sociais (ARS).

Contribuições para a gestão: criação de indicadores de eficiência financeira como ferramentas de apoio à tomada de decisões gerenciais em clubes de futebol.
\end{abstract}

\section{Introdução}

O futebol é considerado o esporte mais popular do mundo (Peeters \& Szymanski, 2014, p. 345) e um grande negócio (Leoncini \& Silva, 2005). Ao longo dos anos, as mudanças ocorridas no futebol levaram os gestores dos clubes a repensar suas formas de gerenciamento financeiro.

Nesse aspecto, Pollard e Reep (1997) consideraram ser difícil pensar a tomada de decisão sem o uso da coleta e análise de dados quantitativos relativos ao esporte. Inerente a isso, investigar o campo esportivo é um desafio, pois envolve concepções microeconômicas relativas aos recursos internos, como jogadores formados e em formação, e macroeconômicas associadas às transferências e redes interorganizacionais da organização esportiva (Booth, 2009). Estender (2014) menciona que o futebol pode ser compreendido como um empreendimento que envolve o mercado de jogadores.

O processo de gestão financeira dos clubes deve proporcionar ciclos sustentáveis em seus saldos, inclusive nos fluxos de jogadores, atentando-se ao valor das despesas feitas no passado e obter valores mais eficientes em suas transações comerciais (Mourão, 2016). Nesse contexto, os clubes profissionais de futebol têm encontrado nas transferências de jogadores uma fonte de equilíbrio nas suas finanças.

$\mathrm{Na}$ gestão do futebol moderno, têm-se as transferências de jogadores que representaram, em média, $16,5 \%$ da receita dos clubes de futebol profissional no Brasil. A gestão ineficiente dessa fonte de receita impacta de forma significativa na questão financeira dos clubes. 
Os estudos relativos à eficiência financeira e ao uso da metodologia de Análise de Redes Sociais (ARS) no futebol vêm ganhando destaque, o que despertou o interesse pelo desenvolvimento da pesquisa relativa eficiência financeira e às transferências em clubes de futebol profissional, conforme destacado por Pina, Paulo e Araújo (2017). Estes autores investigaram a influência da densidade da rede no desempenho da equipe, com ou sem êxito, em 12 jogos da fase de grupos do Grupo $C$ da UEFA Champions League 2015/2016.

Evidências empíricas apresentadas em estudos em clubes de futebol profissional no Brasil, no entanto, sugerem que existe uma ligação entre eficiência financeira e fluxos de jogadores. Em particular, verificou-se que os clubes selecionados têm em comum custo de atletas formados e em formação e receitas operacionais, ceteris paribus.

Com efeito, torna-se importante entender à luz dos indicadores de eficiência financeira, a gestão dos custos com jogadores formados e em formação na geração de receita operacional de clubes que realizaram transferências de jogadores com os quatro clubes semifinalistas do Paulista 2017.

Nesse sentido, este artigo tem o propósito de atingir dois objetivos. No primeiro é apresentar os scores de eficiência financeira de clubes brasileiros que realizaram transferências de jogadores com as equipes semifinalistas do Campeonato Paulista de 2017. O segundo objetivo apresenta os atores e as interações que se destacaram nos fluxos de jogadores junto aos clubes semifinalistas.

Com o intuito de responder aos objetivos, o resto do artigo está organizado da seguinte forma: na seção 2, descreve-se a eficiência financeira e fluxos de jogadores do futebol, enquanto, na seção 3, os procedimentos metodológicos adotados. Na seção 4 apresentam-se os resultados da análise do modelo proposto, as estimativas de eficiência financeira e as redes formadas pelas transferências de jogadores, e por fim, na seção 5 a conclusão.

\section{Eficiência financeira e fluxos de jogadores do futebol}

Os fluxos de jogadores constituem uma das alternativas de aquisição de recursos dos clubes de futebol para formação da equipe e geração de receita operacional. Em particular, nas organizações desportivas, a escassez e o aspecto crítico dos recursos gerenciais desempenham um papel crítico na eficácia organizacional (Penrose, 1959).
Gerrard (2005) apresenta dois aspectos da eficácia organizacional. Em primeiro lugar, a eficácia organizacional requer que o tamanho e a composição do estoque disponível de recursos sejam maximizados em relação às metas de desempenho da organização. Segundo, a eficácia organizacional exige que o nível atingível de desempenho dos resultados seja maximizado (Gerrard, 2005, p. 144). No primeiro aspecto, a eficácia organizacional representa a eficiência alocativa, enquanto o segundo representa eficiência técnica (financeira). Gerrard (2005) assevera que a eficiência financeira é passível de análise estatística transversal nas organizações esportivas, desde que o desempenho organizacional seja controlado por diferenças no uso de seus recursos, como jogadores formados e em formação.

Em relação aos fluxos de jogadores, Frick (2011) afirma que a transferência dos jogadores envolve elementos como salários, aluguéis, direito de propriedade do clube vendedor. Os fluxos dos jogadores de futebol chamam a atenção da academia há décadas (Liu, Liu, Lu, Wang, \& Wang 2016, p. 8). Em relação a esses fluxos do tipo transferência, Liu et al. (2016) afirmaram que podem surgir sub-redes neste âmbito.

A rede de transferências domésticas que contém apenas transferências dentro de uma mesma liga e a rede de transferências internacionais entre diferentes ligas no futebol (Liu, Liu, Lu, Wang, \& Wang, 2016).

Existem diferentes estudos relacionados à eficiência financeira em clubes de futebol. A eficiência financeira relacionada às métricas de redes sociais como a centralidade (entrada e saída), densidade, média de nós e coeficiente de agrupamento em clubes de 24 ligas de classe mundial no período de 2011 a 2015 (Liu, Liu, Lu, Wang, \& Wang, 2016). Os autores concluíram que a eficiência financeira está fortemente associada às características de rede constituídas pelos clubes na transferência de jogadores de futebol.

Os fluxos de jogadores podem também constituir redes inteorganizacionais. Carvalho et al. (2009), ao estudarem uma rede interorganizacional dos atores envolvidos no mercado do futebol concluíram que existia um ator central que comandava toda a rede por meio de seu recurso financeiro. No aspecto financeiro tem-se às receitas (Rohde \& Breuer, 2016), o endividamento (Mourão, 2012) e remuneração de jogadores e duração de contrato (Frick, 2011). 


\section{0}

Eficiência Financeira, Atores e Interações: Um Estudo do Fluxo de Jogadores entre Clubes e as Equipes Semifinalistas de São Paulo em 2017

A análise das redes sociais distinguiu os fluxos de jogadores em empréstimos e transferências, conforme apresentado por Liu et. al. (2016). Na transferência, "o jogador termina seu contrato com o antigo clube e assina um contrato com o novo clube [...]". No empréstimo "o jogador pode jogar temporariamente em um clube diferente do que ele está contratado atualmente" (Liu, Liu, Lu, Wang, \& Wang 2016, p. 8).

$\mathrm{Na}$ análise de redes sociais, as medidas de centralidade constituem ferramentas significativas por contribuírem na compreensão da estrutura da rede do futebol e dos fluxos de jogadores. O Betweenness demonstra o poder como intermediário do ator na rede. Hatala (2006) define que a medida de centralidade se refere à posição de um nó dentro de uma rede particular. A centralidade pode ser vista como um atributo de atores individuais resultante de sua posição na rede (Hanneman \& Riddle, 2005).

\section{Procedimentos Metodológicos}

O objetivo deste artigo é apresentar os scores de eficiência financeira dos clubes brasileiros, os atores e interações que se destacaram nos fluxos de jogadores junto aos clubes semifinalistas. A escolha do lócus de pesquisa se deve ao fato de que o campeonato paulista é considerado a liga de futebol profissional mais antiga do Brasil, criada em 1902.

O levantamento de dados da pesquisa ocorreu em duas etapas. Na primeira etapa foram coletados dados relativos aos clubes envolvidos nos fluxos de jogadores, no período de 2016 e 2017. O primeiro conjunto de dados extraídos do site alemão Transfermarkt continham informações, tais como: nome dos jogadores, clube de origem, clube de destino, agente do futebol, data da entrada, data da saída, país do clube de origem, valor monetário dos fluxos de jogadores no período de 01/01/2016 a $31 / 12 / 2017$.

A segunda etapa da coleta envolveu a extração de dados relativos à receita operacional, fornecedores, custo dos atletas formados e custos dos atletas em formação dos clubes brasileiros. Essas contas foram extraídas do Balanço Patrimonial (BP), do Demonstrativo do Resultado de Exercício (DRE) e das Notas Explicativas de 2016 e 2017.

Adotou-se a escolha dessas variáveis com base nos estudos de Barros, Assaf e Sá-Earp (2009), que utilizam inputs e outputs das contas das demonstrações contábeis de 20 clubes de futebol brasileiros no período 2006 e 2007.

A fronteira estocástica de produção representa a eficiência financeira máxima obtida por um dado de vetor de entrada (Battese \& Coelli, 1995). A combinação de entrada e saída de cada clube está localizada na fronteira de produção com posição acima demonstrando eficiência ou abaixo, ineficiência. Essa fronteira pode, portanto, ser definida a cada momento no tempo na geração de receita operacional dos clubes selecionados.

Para a análise envoltória de dados (DEA) importaram-se os dados para uma matriz do software STATA 14.0. Diante das características dos dados, o modelo mais apropriado para este estudo foi o proposto por Banker, Charnes e Cooper (1984) (BCCVRS), orientado para maximização dos outputs, cujo objetivo é medir a eficiência dos melhores resultados possível, com retornos variáveis na geração de receitas dos clubes. O modelo BCC pressupõe que as unidades de tomada de decisão (DMU's) apresentem retornos variáveis de escala.

A Análise Envoltória de Dados (DEA) é uma técnica que compara as DMUs, que realizam tarefas similares e se diferenciam nas quantidades de inputs que representam os insumos e os outputs que são os produtos (Meza, Neto, Mello, Gomes, \& Coelho, 2005).

Associado a isso, Ji e Lee (2009) afirmaram que o DEA é um método de programação linear não paramétrico que avalia a eficiência e produtividade de unidades produtivas.

Os procedimentos operacionais utilizados na ARS foram: 1ㅇ passo: criação da matriz para interpretação dos dados no UCINET e codificação no bloco de notas para o PAJEK. No caso da análise realizada no UCINET, foi construída a matriz aij, onde a linha e a coluna foram os 69 clubes que realizaram os fluxos de jogadores e também os agentes de futebol. No PAJEK foram criados e codificados os clubes e agentes nos vértices, que representaram o ponto de ligação entre dois edges. Edges foram às linhas que representaram a ligação entre os dois vértices (clubes e agentes). Já os arcos foram às linhas que representaram a ligação entre dois clubes, clubes e agentes, mas indicando a direção, ou seja, clubes de origem e destino, clubes de origem e agentes. 2o Passo: importação da matriz de clubes e agentes para o UCINET e PAJEK. 3 o Passo: análise das métricas de ARS. 40 Passo: interpretação 
e demonstração dos resultados. O uso do PAJEK, complementando o UCINET, se deve à necessidade de demonstrar a quantidade de fluxos entre os atores.

Nos fluxos de jogadores estudados neste artigo, os nós são as equipes que realizaram as transferências de jogadores no período selecionado, os agentes de futebol e as arestas direcionadas, que conectam os nós, são os fluxos de jogadores.

Nas estimativas de atores centrais optou-se pela medida de Betweenness e centralidade. $\mathrm{Na}$ identificação dos clubes que mais transferiram ou emprestaram jogadores, foi utilizada a métrica do grau de centralidade.

$\mathrm{Na}$ próxima seção serão apresentados os resultados do modelo testado, as estimativas dos fluxos de jogadores e a eficiência financeira dos clubes analisados.

\section{Descrição dos Resultados}

Nesta seção serão apresentados os resultados dos testes do modelo proposto e os scores de eficiência financeira dos clubes profissionais de futebol.
Também são apresentados os atores e as interações que se destacaram nos fluxos jogadores junto aos clubes semifinalistas.

\section{O modelo de eficiência financeira}

A regressão linear multivariada é utilizada para investigar dependência entre uma variável dependente (métrica) e variáveis independentes (Prearo, Gouvêa, \& Romeiro, 2012). Com efeito, realizou-se o processo de avaliação do modelo proposto, por meio da regressão multivariada no software Statistical Package for Social Science for Windows (SPSS 22.0).

A robustez do modelo é demonstrada a partir das premissas subjacentes ao uso da técnica de Regressão Linear Multivariada pelo nível de significância (t de Student), teste de multicolinearidade (VIF), autocorrelação de resíduos (Durbin-Watson), homoscedasticidade (teste White) e tamanho da amostra.

Os resultados demonstraram que as variáveis independentes CAF e CAFOR são estatisticamente significativas a $1 \%$ (Figura 1 ).

\begin{tabular}{|c|c|c|c|}
\hline Premissas & Teste & Resultado & Conclusão \\
\hline Nível de significância & $t$ de Student & $\begin{array}{l}\text { CAF } p \text {-valor } 0,0094 * * * \\
\text { CAFOR } p \text {-valor } 0,0003 * * * \\
\text { FOR } p \text {-valor } 0,882\end{array}$ & $\begin{array}{l}\text { Significativo a } 1 \% \\
\text { Significativo a } 1 \% \\
\text { Sem significância }\end{array}$ \\
\hline Baixa multicolinearidade & VIF & $\begin{array}{l}\text { CAF }(\mathrm{VIF}=2,317<5) \\
\text { CAFOR }(\mathrm{VIF}=1,600<5)\end{array}$ & $\begin{array}{ll}\text { Sem indícios } & \text { de } \\
\text { multicolinearidade } & \end{array}$ \\
\hline $\begin{array}{l}\text { Ausência de erros } \\
\text { correlacionados }\end{array}$ & $\begin{array}{l}\text { Durbin- } \\
\text { Watson }\end{array}$ & $2,139352>2$ & $\begin{array}{l}\text { Ausência de autocorrelação } \\
\text { dos resíduos }\end{array}$ \\
\hline Homoscedasticidade & $\begin{array}{l}\text { Teste de } \\
\text { White }\end{array}$ & $\begin{array}{l}\text { Hipótese nula: } \\
\text { heteroscedasticidade }\end{array}$ & $\begin{array}{l}\text { Estatística de teste: } \mathrm{LM}= \\
8,27869 \text { com } \mathrm{p} \text {-valor }=\mathrm{P}(\text { Qui- } \\
\text { quadrado(5) > 8,27869) }= \\
0,141531\end{array}$ \\
\hline Tamanho da amostra & $\begin{array}{l}>5 \text { casos por } \\
\text { variável }\end{array}$ & 3 variáveis, com 42 clubes & Mais de 5 casos por variável \\
\hline
\end{tabular}

Figura 1. Premissas subjacentes ao uso das técnicas de regressão linear múltipla

Fonte: Hair, Anderson, Tatham, \& Black ( 2005) e Prearo, Gouvêa, \& Romeiro (2012)

O modelo de explicação apresentou um $R^{2}$ (ajustado) de 0,602116, indicando que 60,2\% das variações da receita operacional (em milhares de reais) são explicadas pelas variáveis independentes. A reta de regressão foi definida como:

$Y_{i t}=20.423 .625,538+3,05606 \mathrm{CAF}_{i t}+1,21792$ CAFOR $_{\text {it }}$.

Onde,

a subseção $i(i=1,2, \ldots, n)$ refere-se ao clube da amostra e o índice $t(t=1,2,3)$ refere-se ao ano $t$;

$$
Y=\text { denota do total da Receita Operacional dos Clubes }
$$
Brasileiros;

$\mathrm{CAF}=$ denota o valor do Custo de Atleta em Formação; CAFOR = denota o valor do Custo de Atleta Formado.

A reta de regressão indicou que a média da receita operacional dos clubes está em torno de R\$ 20.423.625,538. A regressão demonstrou uma relação positiva e significativa a $1 \%$ entre as variáveis 


\section{2}

Eficiência Financeira, Atores e Interações: Um Estudo do Fluxo de Jogadores entre Clubes e as Equipes Semifinalistas de São Paulo em 2017

dos custos de atletas formados e em formação com a receita operacional.

A Receita Operacional envolve o valor total das contas como direitos de transmissão de TV, patrocínios e publicidades, premiações, fiel torcedor, loterias, receitas com repasses de direitos federativos, receita de jogos, negociação de atletas (transferências e empréstimos), locações, licença de logomarca, promoções e eventos, indenizações, ensino e educação, entre outras.

Os Custos de Atletas em Formação incluem os gastos incorridos com os atletas em formação (ou das categorias de base), atribuídos individualmente aos atletas ainda não profissionalizados.

Os Custos de Atletas Profissionais Formados constituem os gastos com contratações de atletas no mercado ou profissionalizados oriundos das categorias de base, estando os contratos em vigor ao final do exercício.
No processo de escolha de outra técnica não paramétrica, realizou-se o teste de normalidade dos dados da receita operacional dos clubes de futebol. A hipótese testada afirmava que o comportamento da variável receita operacional possui um comportamento normal. O teste Shapiro-Wilk apresentou um valor de $p$-valor $=0,000<0,05$, demonstrando uma distribuição não normal da variável observada e indicando testes não paramétricos.

\section{Eficiência financeira dos clubes brasileiros de futebol}

Com o intuito de responder ao primeiro objetivo, na Tabela 1 são apresentados os clubes brasileiros que realizaram transferências de jogadores com as equipes semifinalistas de 2017.

Na Tabela 1 são comentadas as variáveis utilizadas no modelo financeiro, tendo como vetores de entrada os custos dos atletas em formação e formados na geração da receita operacional.

Tabela 1. Variáveis utilizadas no modelo financeiro "em milhares de R\$̣ (reais)" em 2016

\begin{tabular}{|c|c|c|c|}
\hline \multirow[b]{2}{*}{ Clube } & \multirow{2}{*}{$\begin{array}{c}\text { Output } \\
\text { Receita Operacional }\end{array}$} & \multicolumn{2}{|c|}{ Inputs } \\
\hline & & Custo do Atleta em Formação & Custo do Atleta Formado \\
\hline Atlético-MG & 316.312 & 7.242 & 84.280 \\
\hline Atlético-PR & 126.329 & 13.357 & 61.345 \\
\hline Bahia & 112.943 & 21.190 & 6.584 \\
\hline Chapecoense & 64.433 & 2.833 & 28.187 \\
\hline Corinthians & 433.903 & 83.337 & 123.980 \\
\hline Coritiba & 102.210 & 22.015 & 53.966 \\
\hline Criciúma & 25.964 & 4.968 & 15.125 \\
\hline Cruzeiro & 222.404 & 20.408 & 149.267 \\
\hline Figueirense & 70.891 & 3.984 & 36.189 \\
\hline Fluminense & 271.896 & 5.850 & 121.731 \\
\hline Grêmio & 212.662 & 33.024 & 190.241 \\
\hline Internacional & 266.861 & 9.143 & 88.812 \\
\hline Joinville & 29.119 & 2.218 & 21.888 \\
\hline Palmeiras & 477.502 & 15.973 & 3.784 \\
\hline Paraná & 13.483 & 5.631 & 195 \\
\hline Ponte Preta & 64.579 & 25.130 & 59.610 \\
\hline São Paulo & 337.213 & 40.734 & 80.453 \\
\hline Sport & 108.248 & 5.690 & 5.690 \\
\hline Vasco & 204.654 & 55.128 & 13.002 \\
\hline Vila Nova & 12.925 & 3.720 & 4.784 \\
\hline Vitória & 111.976 & 5.451 & 104.648 \\
\hline
\end{tabular}

Fonte: elaborado pelos autores a partir das demonstrações contábeis (2018) 


\section{3}

E. E. Silva, A. A. Santos, M. A. P. Silveira, P. J. R. Mourão

Na Tabela 2 são apresentadas as variáveis relativas à receita operacional em 2017.

Tabela 2. Variáveis utilizadas no modelo financeiro "em milhares de R\$ (reais)" em 2017

\begin{tabular}{|c|c|c|c|}
\hline \multirow[b]{2}{*}{ Clube } & \multirow{2}{*}{$\begin{array}{c}\text { Output } \\
\text { Receita Operacional }\end{array}$} & \multicolumn{2}{|c|}{ Input } \\
\hline & & Custo do Atleta em Formação & Custo do Atleta Formado \\
\hline Atlético-MG & 311.365 & 8.463 & 95.022 \\
\hline Atlético-PR & 130.667 & 16.257 & 62.050 \\
\hline Bahia & 97.083 & 19.644 & 16.447 \\
\hline Chapecoense & 94.545 & 4.173 & 48.593 \\
\hline Corinthians & 358.105 & 80.607 & 158.687 \\
\hline Coritiba & 111.861 & 19.091 & 62.304 \\
\hline Criciúma & 17.386 & 4.149 & 14.332 \\
\hline Cruzeiro & 283.379 & 27.886 & 148.553 \\
\hline Figueirense & 26.533 & 3.896 & 20.263 \\
\hline Fluminense & 212.156 & 6.779 & 115.460 \\
\hline Grêmio & 322.581 & 14.319 & 250.171 \\
\hline Internacional & 228.012 & 10.222 & 108.086 \\
\hline Joinville & 17.875 & 1.505 & 12.743 \\
\hline Palmeiras & 503.682 & 15.780 & 3.569 \\
\hline Paraná & 22.570 & 3.552 & 806 \\
\hline Ponte Preta & 68.768 & 39.984 & 42.000 \\
\hline São Paulo & 423.716 & 38.431 & 106.611 \\
\hline Sport & 71.511 & 4.299 & 4.299 \\
\hline Vasco & 185.691 & 10.407 & 29.738 \\
\hline Vila Nova & 37.956 & 3.721 & 31.235 \\
\hline Vitória & 88.071 & 6.837 & 82.485 \\
\hline
\end{tabular}

Fonte: elaborado pelos autores a partir das demonstrações contábeis (2018)

Na Tabela 3 são apresentados os scores de eficiência financeira (DEA) dos clubes brasileiros, no biênio de 2016 e 2017. Nos anos de 2016 e 2017, entre os quatro semifinalistas, somente o Palmeiras demonstrou eficiência na geração de receita operacional com o uso dos recursos de custos dos atletas em formação e atletas formados. Evidencia-se que, o Clube Atlético Mineiro também foi eficiente financeiramente nos dois períodos. O Fluminense apresentou eficiência financeira em 2016. 


\section{4}

Eficiência Financeira, Atores e Interações: Um Estudo do Fluxo de Jogadores entre Clubes e as Equipes Semifinalistas de São Paulo em 2017

Tabela 3. Resultado dos scores de eficiência financeira dos clubes brasileiros

\begin{tabular}{|c|c|c|c|}
\hline & & 2016 & 2017 \\
\hline $\mathrm{N}$ & Clube & E-DEA & E-DEA \\
\hline 1 & Atlético-MG & 1,00 & 1,00 \\
\hline 2 & Atlético-PR & 0,26 & 0,23 \\
\hline 3 & Bahia & 0,17 & 0,15 \\
\hline 4 & Chapecoense & 0,54 & 0,61 \\
\hline 5 & Corinthians & 0,16 & 0,13 \\
\hline 6 & Coritiba & 0,14 & 0,17 \\
\hline 7 & Criciúma & 0,15 & 0,12 \\
\hline 8 & Cruzeiro & 0,28 & 0,29 \\
\hline 9 & Figueirense & 0,43 & 0,19 \\
\hline 10 & Fluminense & 1,00 & 0,85 \\
\hline 11 & Grêmio & 0,17 & 0,61 \\
\hline 12 & Internacional & 0,70 & 0,61 \\
\hline 13 & Joinville & 0,31 & 0,33 \\
\hline 14 & Palmeiras & 1,00 & 1,00 \\
\hline 15 & Paraná & 0,54 & 0,19 \\
\hline 16 & Ponte Preta & 0,07 & 0,05 \\
\hline 17 & São Paulo & 0,25 & 0,33 \\
\hline 18 & Sport & 0,61 & 0,51 \\
\hline 19 & Vasco & 0,12 & 0,53 \\
\hline 20 & Vila Nova & 0,11 & 0,28 \\
\hline 21 & Vitória & 0,44 & 0,35 \\
\hline
\end{tabular}

Fonte: elaborado pelos autores a partir das demonstrações contábeis (2018)

Os fluxos de jogadores contribuem na aquisição de recursos dos clubes de futebol, sendo assim, a visualização dos fluxos unidirecionais, ou seja, de única direção (Hanneman \& Riddle, 2005), das múltiplas relações estabelecidas pelos clubes de futebol são exploradas na seção a seguir.

\section{Atores e interação nos fluxos de jogadores nos clubes}

As transações comerciais (Mourão, 2016) realizadas pelos clubes selecionados constituem instrumentos de aquisição de recursos financeiros. Os fluxos de jogadores representam, em média, $16,5 \%$ da receita dos clubes de futebol profissional no Brasil, conforme demonstrado na Figura 2.

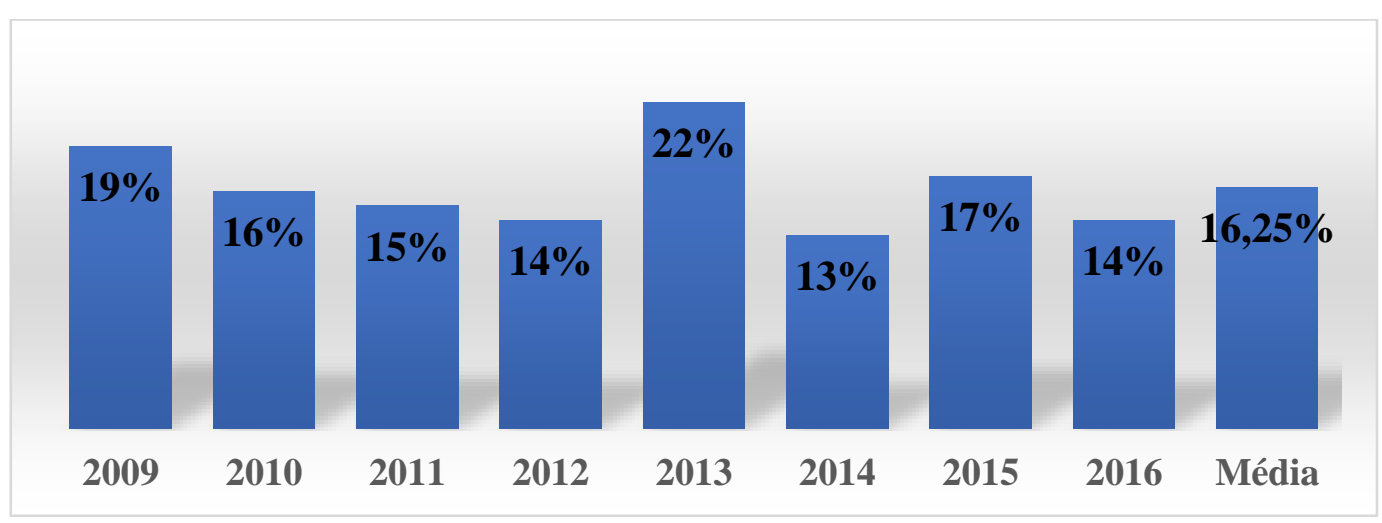

Figura 2. Percentual de receitas de movimentações de jogadores no Brasil de 2009 a 2016 Fonte: elaborados a partir do Transfermarkt (2018) 
A visualização dos fluxos de jogadores pode ser observada na Figura 2, indicando as múltiplas relações estabelecidas pelos clubes de futebol. A Figura 2 apresentou um fluxo unidirecional (Hanneman \& Riddle, 2005), onde há interação de um par de atores envolvidos na relação num simples segmento de linha, sem ponta de seta formada pelos quatro clubes semifinalistas do Paulista 2017.
A Figura 3 possibilitou identificar, de maneira visual, os fluxos de jogadores dos clubes e as equipes semifinalistas. Os clubes de origem dos fluxos jogadores estão representados pelo símbolo "quadrado" na cor azul. Os clubes de destino dos fluxos de jogadores estão retratados pelo símbolo "quadrado" e nas cores vermelha, verde, branca e preta.

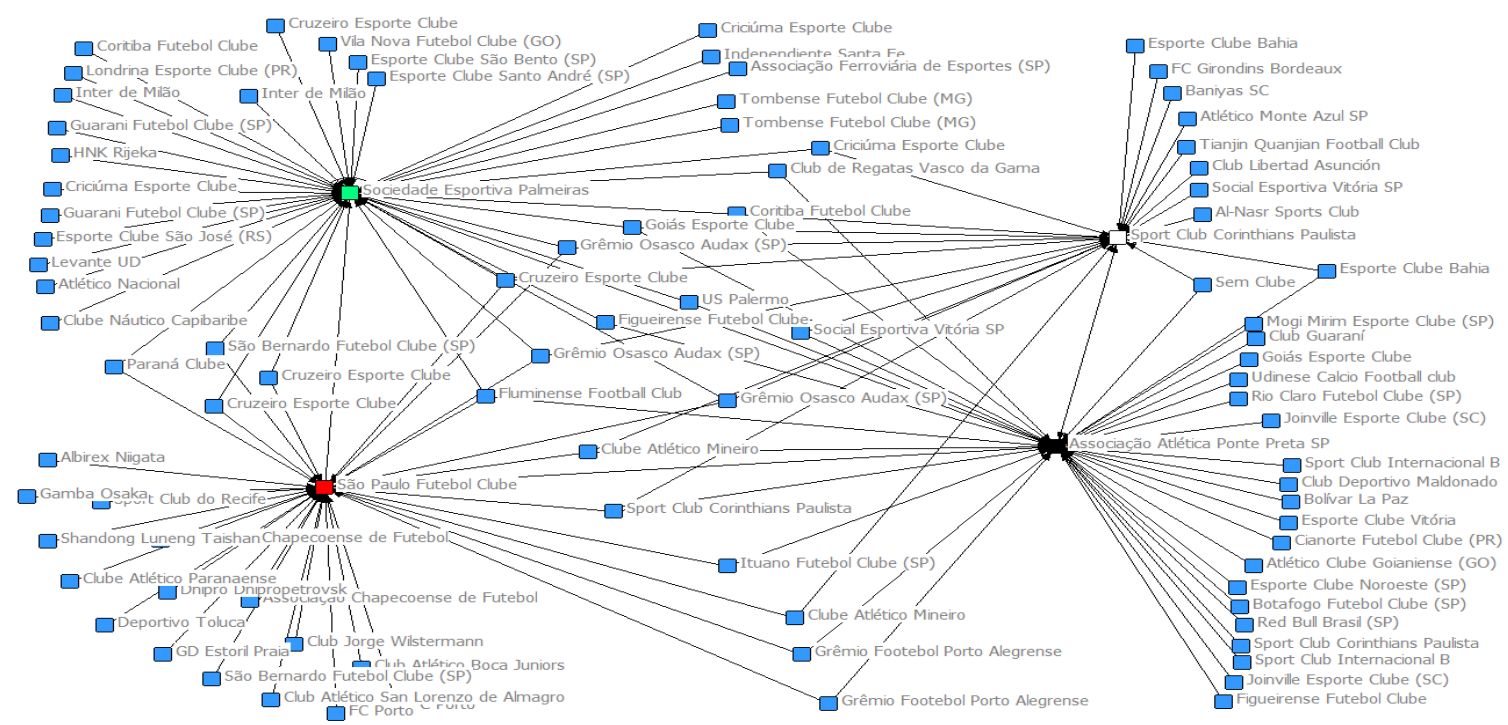

Figura 3. Grafo das relações de fluxos de jogadores nos clubes selecionados

Fonte: elaboração dos autores a partir do Transfermarkt (2018)

Evidencia-se que existem 128 fluxos de jogadores do futebol, conforme demonstrado na Tabela 3. entre os clubes selecionados com diferentes agentes

Tabela 3. Agentes envolvidos nos fluxos de jogadores dos clubes selecionados

\begin{tabular}{|c|c|c|c|c|c|}
\hline \multirow[b]{2}{*}{ Agente } & \multicolumn{4}{|c|}{ Clubes Semifinalistas } & \multirow[b]{2}{*}{ Total } \\
\hline & Corinthians & Ponte Preta SP & São Paulo & Palmeiras & \\
\hline Sem agente & 7 & 12 & 14 & 14 & 47 \\
\hline Elenko Sports Ltda. & 2 & 4 & 0 & 0 & 6 \\
\hline Marco Kirdemir & 1 & 1 & 0 & 0 & 2 \\
\hline AGN R2M Football & 1 & 0 & 0 & 0 & 1 \\
\hline Carlos Leite & 2 & 0 & 0 & 0 & 2 \\
\hline Think Ball \& Sports Consulting & 1 & 0 & 0 & 0 & 1 \\
\hline Planet Soccer & 0 & 1 & 0 & 0 & 1 \\
\hline Eduardo Uram & 0 & 2 & 2 & 1 & 5 \\
\hline $\begin{array}{l}\text { Pro Soccer MGMT } \\
\text { Intermediações }\end{array}$ & 0 & 1 & 0 & 0 & 1 \\
\hline Souza Gutemberg & 0 & 1 & 0 & 0 & 1 \\
\hline Samii Sport [...] & 0 & 1 & 1 & 0 & 2 \\
\hline Sport Base Brasil & 0 & 1 & 0 & 1 & 2 \\
\hline Exclusive Sports GbR & 0 & 1 & 0 & 0 & 1 \\
\hline Showball & 0 & 1 & 0 & 0 & 1 \\
\hline Groupe USM & 0 & 1 & 0 & 0 & 1 \\
\hline MFD Sports & 0 & 2 & 0 & 0 & 2 \\
\hline Monte Areo Sports & 0 & 1 & 0 & 0 & 1 \\
\hline
\end{tabular}




\section{6}

Eficiência Financeira, Atores e Interações: Um Estudo do Fluxo de Jogadores entre Clubes e as Equipes Semifinalistas de São Paulo em 2017

\begin{tabular}{|c|c|c|c|c|c|}
\hline Europe Sports Group & 2 & 2 & 2 & 2 & 8 \\
\hline Gortin Promoções Ltda & 0 & 1 & 1 & 0 & 2 \\
\hline WHK & 0 & 1 & 0 & 0 & 1 \\
\hline Soccertalents & 0 & 1 & 1 & 0 & 2 \\
\hline Un1que football & 0 & 1 & 1 & 5 & 7 \\
\hline ACRsoccer - Sports [...] & 1 & 0 & 0 & 0 & 1 \\
\hline Marcio Bittencourt Sports & 1 & 0 & 0 & 0 & 1 \\
\hline AllemanhaBrasil & 1 & 0 & 1 & 0 & 2 \\
\hline Relatives & 1 & 0 & 0 & 0 & 1 \\
\hline Grupo Empenho & 1 & 0 & 0 & 0 & 1 \\
\hline Elite Consulting Europe & 0 & 0 & 1 & 0 & 1 \\
\hline Firsteleven ISM & 0 & 0 & 1 & 0 & 1 \\
\hline New Idea Sports \& Marketing & 0 & 0 & 1 & 0 & 1 \\
\hline Onsoccer & 0 & 0 & 1 & 0 & 1 \\
\hline HWS Business Intermediations & 0 & 0 & 1 & 0 & 1 \\
\hline Familiar & 0 & 0 & 0 & 1 & 1 \\
\hline Magnitude Sports \& [...] & 0 & 0 & 1 & 0 & 1 \\
\hline Sbsfoot & 0 & 0 & 0 & 1 & 1 \\
\hline Velop4 Sports Ltda & 0 & 0 & 0 & 1 & 1 \\
\hline Luiz Taveira & 0 & 0 & 0 & 1 & 1 \\
\hline GMRsports & 0 & 0 & 0 & 1 & 1 \\
\hline Footinvest Managemet-S.A & 0 & 0 & 0 & 4 & 4 \\
\hline Promoesport & 0 & 0 & 0 & 2 & 2 \\
\hline World Soccer Star GMBH & 0 & 0 & 0 & 1 & 1 \\
\hline Football Capital & 0 & 0 & 0 & 1 & 1 \\
\hline World Sportbusiness & 0 & 0 & 0 & 1 & 1 \\
\hline Star Player GROUP & 0 & 0 & 0 & 1 & 1 \\
\hline MANAGERfoot & 0 & 0 & 0 & 1 & 1 \\
\hline Pro Futebol & 0 & 0 & 2 & 0 & 2 \\
\hline $\mathrm{B} 2 \mathrm{~F}$ & 1 & 0 & 0 & 0 & 1 \\
\hline Total & 22 & 36 & 31 & 39 & 128 \\
\hline
\end{tabular}

Fonte: elaborado a partir do transfermarkt (2018)

Observa-se pela Tabela 3, que a Sociedade Esportiva Palmeiras realizou o maior número de fluxos de jogadores, seguido pela Associação Atlética Ponte Preta, São Paulo Futebol Clube e Sport Club Corinthians Paulista.
Na Tabela 4 são demonstrados os valores das transferências realizadas no período de 2016 e 2017 dos clubes paulistas.

Tabela 4. Valores monetários das transferências de jogadores (2016-2017)

\begin{tabular}{lll}
\hline Clube & Transferência & Valor Monetário (€) \\
\hline Associação Atlética Ponte Preta SP & 3 & $800.000,00$ \\
São Paulo Futebol Clube & 10 & $24.215 .000,00$ \\
Sociedade Esportiva Palmeiras & 13 & $36.533 .000,00$ \\
Sport Clube Corinthians Paulista & 8 & $11.725 .000,00$ \\
\hline
\end{tabular}

Fonte: elaboração dos autores a partir do Transfermarkt (2018)

Na Tabela 5 são evidenciados os diferentes fluxos de jogadores realizados pelos clubes semifinalistas do Paulista 2017. Observa-se que os fluxos do tipo empréstimo foi o que mais ocorreu no período analisado 
Tabela 5. Fluxos de jogadores dos clubes semifinalistas (2016-2017)

\begin{tabular}{l|c|c|c|c|c}
\hline \multirow{2}{*}{\multicolumn{1}{c}{ Tipo de Movimentação }} & \multicolumn{4}{c|}{ Clube de destino } & \multirow{2}{*}{ Total } \\
\cline { 2 - 6 } & Corinthians & Ponte Preta & São Paulo & Palmeiras & 18 \\
\hline Sem Custo ou custo zero & 0 & 12 & 3 & 3 & 34 \\
\hline Com custo & 8 & 3 & 10 & 20 & 59 \\
\hline Empréstimo & 8 & 13 & 18 & 0 & 4 \\
\hline Transferência livre & 4 & 0 & 0 & 2 & 11 \\
\hline Não declarado & 2 & 7 & 0 & 1 & 2 \\
\hline Total & 0 & 1 & 0 & 31 & 39 \\
\hline
\end{tabular}

Fonte: elaboração dos autores a partir do Transfermarkt (2018)

Na Figura 4 são apresentadas as relações entre agentes e clubes. Os agentes dos jogadores são representados pelo círculo na cor vermelha e os clubes de destino são representados pelo quadrado na cor azul, dos fluxos de jogadores numa única direção (Hanneman \& Riddle, 2005).

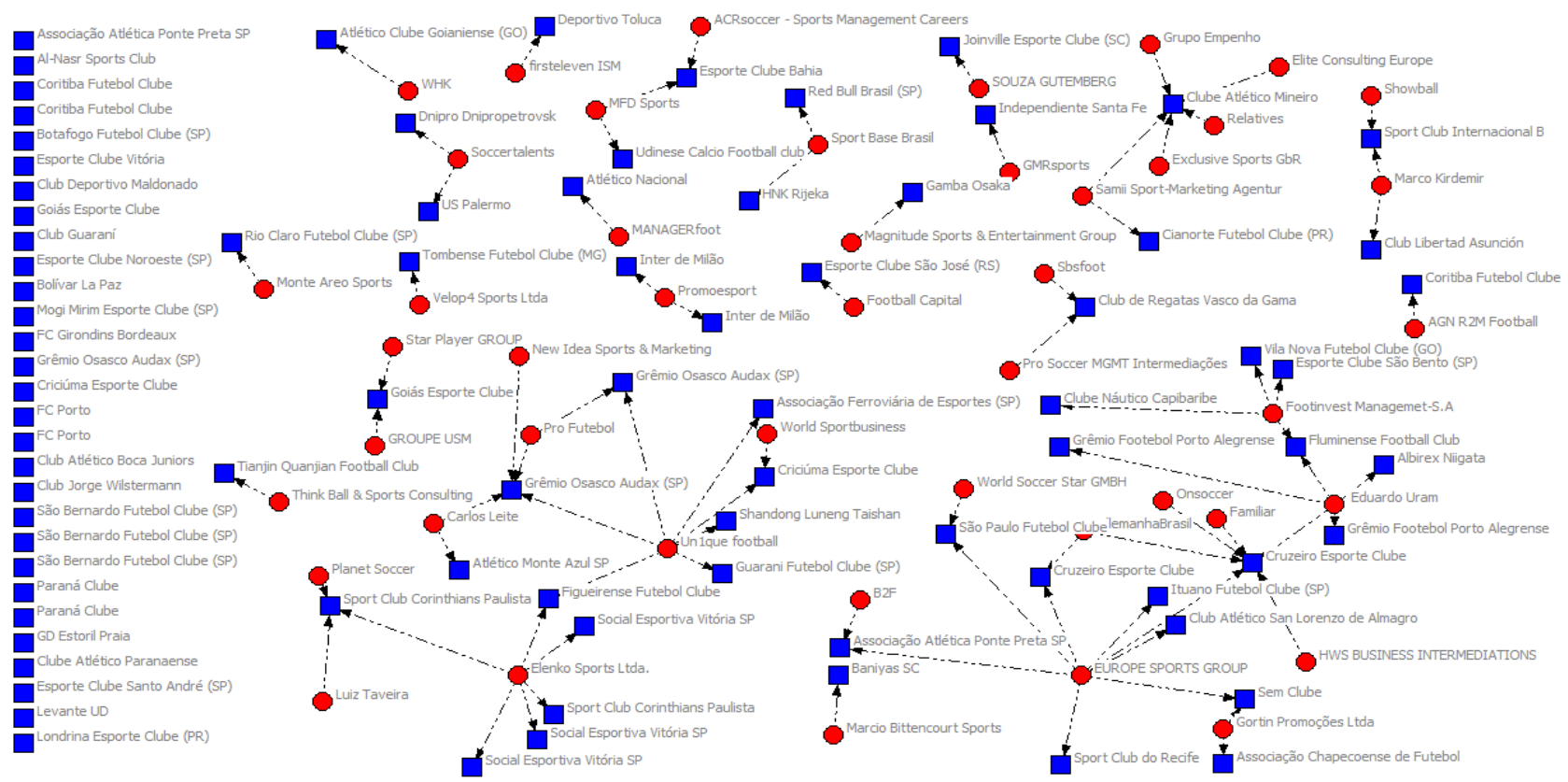

Figura 4. Grafo dos agentes de futebol na rede de negócios Fonte: elaboração dos autores a partir do Transfermarkt (2018)

Observa-se que os agentes que se destacaram nos fluxos de jogadores apareceram com uma medida do betweenness acima de 0 (Tabela 6). Essa métrica demonstrou o poder de intermediação do ator na promoção dos fluxos de jogadores entre os clubes de futebol (Hatala, 2006). 


\section{8}

Eficiência Financeira, Atores e Interações: Um Estudo do Fluxo de Jogadores entre Clubes e as Equipes Semifinalistas de São Paulo em 2017

Tabela 6. Agentes do futebol com poderes na rede de semifinalistas do Paulista 2017

\begin{tabular}{lll}
\hline$N$ & Ator & Betweenness \\
\hline 1 & EUROPE SPORTS GROUP & 0.024 \\
\hline 2 & Eduardo Uram & 0.019 \\
\hline 3 & Um1que football & 0.017 \\
\hline 4 & Elenko Sports Ltda. & 0.013 \\
\hline 5 & Footinvest Managemet-S.A & 0.008 \\
\hline 7 & Gortin Promoções Ltda & 0.003 \\
\hline 8 & Carlos Leite & 0.002 \\
\hline 9 & Samii Sport-Marketing Agentur & 0.001 \\
\hline 10 & AllemanhaBrasil & 0.001 \\
\hline Fonte: & Demais atores & 0.000 \\
\hline
\end{tabular}

Fonte: elaboração dos autores a partir do Transfermarkt (2018)

Na Tabela 7 são demonstrados os países dos clubes de futebol que participaram dos fluxos de jogadores com os clubes semifinalistas do paulista. Observa-se que os fluxos de jogadores, em sua maioria (78,9\%) deram ênfase aos fluxos domésticos, ou seja, com clubes do Brasil. Nos fluxos de jogadores entre clubes brasileiros e de outros países se destacaram equipes da Itália e Portugal.

Tabela 7. Rede de fluxos de jogadores por país de origem dos clubes

\begin{tabular}{l|c|c|l|c|c}
\hline País & Freq. & $(\%)$ & País & Freq. & $(\%)$ \\
\hline Brazil & 101 & 78,9 & Colombia & 2 & 1,6 \\
\hline Italy & 5 & 3,9 & United Arab Emirates & 1 & 0,8 \\
\hline Portugal & 3 & 2,3 & Uruguay & 1 & 0,8 \\
\hline China & 2 & 1,6 & Mexico & 1 & 0,8 \\
\hline Bolívia & 2 & 1,6 & Ukraine & 1 & 0,8 \\
\hline Paraguay & 2 & 1,6 & Croatian & 1 & 0,8 \\
\hline Argentina & 2 & 1,6 & Spain & 1 & 0,8 \\
\hline Japan & 2 & 1,6 & France & 128 & 0,8 \\
\hline Total & & & 100,0 \\
\hline
\end{tabular}

Fonte: elaboração dos autores a partir do Transfermarkt (2018)

Com o objetivo de identificar as equipes que mais realizaram fluxos de jogadores, têm-se as métricas do grau de entrada (indegree) e grau de saída (outdegree).

No que tange aos clubes que cederam ou receberam tem-se a métrica de centralidade de grau (Centrality
Degree) (Hanneman \& Riddle, 2005). Na Figura 5 são demonstrados que os clubes que mais cederam jogadores foram o Grêmio Osasco Audax e Cruzeiro Esporte Clube, respectivamente. 


\begin{tabular}{|c|c|c|c|c|c|}
\hline Clubes & Saída & Entrada & Clubes & Saída & Entrada \\
\hline Grêmio Osasco Audax (SP) & 9.000 & 0.000 & Bolívar La Paz & 1.000 & 0.000 \\
\hline Cruzeiro Esporte Clube & 8.000 & 0.000 & Botafogo Futebol Clube (SP) & 1.000 & 0.000 \\
\hline Clube Atlético Mineiro & 5.000 & 0.000 & Cianorte Futebol Clube (PR) & 1.000 & 0.000 \\
\hline Criciúma Esporte Clube & 5.000 & 0.000 & Club Atlético Boca Juniors & 1.000 & 0.000 \\
\hline Sport Club Corinthians Paulista & 5.000 & 22.000 & $\begin{array}{l}\text { Club Atlético San Lorenzo de } \\
\text { Almagro }\end{array}$ & 1.000 & 0.000 \\
\hline Coritiba Futebol Clube & 4.000 & 0.000 & Club Deportivo Maldonado & 1.000 & 0.000 \\
\hline Goiás Esporte Clube & 4.000 & 0.000 & Club Guaraní & 1.000 & 0.000 \\
\hline Grêmio Footebol Porto Alegrense & 4.000 & 0.000 & Club Jorge Wilstermann & 1.000 & 0.000 \\
\hline Esporte Clube Bahia & 3.000 & 0.000 & Club Libertad Asunción & 1.000 & 0.000 \\
\hline Figueirense Futebol Clube & 3.000 & 0.000 & Clube Atlético Paranaense & 1.000 & 0.000 \\
\hline Fluminense Football Club & 3.000 & 0.000 & Clube Náutico Capibaribe & 1.000 & 0.000 \\
\hline São Bernardo Futebol Clube (SP) & 3.000 & 0.000 & Deportivo Toluca & 1.000 & 0.000 \\
\hline Social Esportiva Vitória SP & 3.000 & 0.000 & Dnipro Dnipropetrovsk & 1.000 & 0.000 \\
\hline Associação Atlética Ponte Preta SP & 2.000 & 36.000 & Esporte Clube Noroeste (SP) & 1.000 & 0.000 \\
\hline $\begin{array}{l}\text { Associação Chapecoense de } \\
\text { Futebol }\end{array}$ & 2.000 & 0.000 & $\begin{array}{l}\text { Esporte Clube Santo André } \\
\text { (SP) }\end{array}$ & 1.000 & 0.000 \\
\hline Club de Regatas Vasco da Gama & 2.000 & 0.000 & Esporte Clube São Bento (SP) & 1.000 & 0.000 \\
\hline FC Porto & 2.000 & 0.000 & Esporte Clube São José (RS) & 1.000 & 0.000 \\
\hline Guarani Futebol Clube (SP) & 2.000 & 0.000 & Esporte Clube Vitória & 1.000 & 0.000 \\
\hline Inter de Milão & 2.000 & 0.000 & FC Girondins Bordeaux & 1.000 & 0.000 \\
\hline Ituano Futebol Clube (SP) & 2.000 & 0.000 & Gamba Osaka & 1.000 & 0.000 \\
\hline Joinville Esporte Clube (SC) & 2.000 & 0.000 & GD Estoril Praia & 1.000 & 0.000 \\
\hline Paraná Clube & 2.000 & 0.000 & HNK Rijeka & 1.000 & 0.000 \\
\hline São Paulo Futebol Clube & 2.000 & 31.00 & Independiente Santa Fe & 1.000 & 0.000 \\
\hline Sem Clube & 2.000 & 0.000 & Levante UD & 1.000 & 0.000 \\
\hline Sport Club Internacional B & 2.000 & 0.000 & Londrina Esporte Clube (PR) & 1.000 & 0.000 \\
\hline Tombense Futebol Clube (MG) & 2.000 & 0.000 & $\begin{array}{l}\text { Mogi Mirim Esporte Clube } \\
\text { (SP) }\end{array}$ & 1.000 & 0.000 \\
\hline US Palermo & 2.000 & 0.000 & Red Bull Brasil (SP) & 1.000 & 0.000 \\
\hline Albirex Niigata & 1.000 & 0.000 & Rio Claro Futebol Clube (SP) & 1.000 & 0.000 \\
\hline Al-Nasr Sports Club & 1.000 & 0.000 & Shandong Luneng Taishan & 1.000 & 0.000 \\
\hline $\begin{array}{l}\text { Associação Ferroviária de Esportes } \\
\text { (SP) }\end{array}$ & 1.000 & 0.000 & $\begin{array}{l}\text { Sociedade Esportiva } \\
\text { Palmeiras }\end{array}$ & 1.000 & 38.00 \\
\hline Atlético Clube Goianiense (GO) & 1.000 & 0.000 & Sport Club do Recife & 1.000 & 0.000 \\
\hline Atlético Monte Azul SP & 1.000 & 0.000 & $\begin{array}{l}\text { Tianjin Quanjian Football } \\
\text { Club }\end{array}$ & 1.000 & 0.000 \\
\hline Atlético Nacional & 1.000 & 0.000 & Udinese Calcio Football Club & 1.000 & 0.000 \\
\hline Baniyas SC & 1.000 & 0.000 & Vila Nova Futebol Clube (GO) & 1.000 & 0.000 \\
\hline
\end{tabular}

Figura 5. Graus de saída e entrada dos clubes da amostra, em 2016 e 2017

Fonte: elaboração dos autores a partir do Transfermarkt (2018)

Na Figura 5 são demonstrados que 13 clubes realizaram no mínimo três fluxos de jogadores. Pelos dados da pesquisa pode-se inferir que a Sociedade Esportiva Palmeiras foi o clube paulista que mais realizou fluxos de jogadores do período analisado.

\section{Conclusão}

Os scores de eficiência financeira forneceram evidências de que entre os clubes semifinalistas do paulista, o Palmeiras foi o único que apresentou eficiência máxima no uso dos recursos de custos de atletas em formação e atletas formados na geração de receita operacional.

Entre os clubes brasileiros tem-se o Clube Atlético Mineiro que também demonstrou eficiência financeira máxima em 2016 e 2017 e o Fluminense somente em 2016. 


\section{0}

Eficiência Financeira, Atores e Interações: Um Estudo do Fluxo de Jogadores entre Clubes e as Equipes Semifinalistas de São Paulo em 2017

Os atores que se destacaram como intermediadores nos fluxos de jogadores junto aos clubes e as equipes semifinalistas do paulista de 2017 foram os agentes Europe Sports Group, Eduardo Uram, Un1que Football e Elenko Sports Ltda.

Dentre os clubes semifinalistas, o Palmeiras realizou o maior número de fluxos de jogadores, seguido pela Ponte Preta, São Paulo e Corinthians, respectivamente. O Palmeiras e o São Paulo utilizaram empréstimos e transferências de jogadores, a Ponte Preta as negociações sem custo ou custo zero. O Corinthians realizou empréstimos e transferências. Os clubes que mais cederam jogadores para os clubes semifinalistas foram o Grêmio Osasco Audax e o Cruzeiro Esporte Clube, respectivamente.

Em relação às interações dos fluxos de jogadores ocorreram em sua maioria por meio de empréstimos, entre clubes do Brasil. Nos fluxos de jogadores entre clubes brasileiros e de outros países se destacaram as equipes da Itália e Portugal.

A análise da fronteira estocástica contribuiu para demonstrar os clubes com scores de eficiência máximo no uso de recursos de atletas em formação e atletas formados na geração de receita operacional. Os resultados de maiores scores eficiência forneceram evidências do nível máximo de

\section{Referências}

Banker, R. D., Charnes, A., \& Coopers, A. A. (1984). "Some Models for Estimating Technical and Scale Inefficiencies in Data Envelopment Analysis", Management Science,30, (9), pp.1078-1092.

Barros, C. P., Assaf, A., \& Sá-Earp, F. (2009). Brazilian Football Technical Efficiency: A Bootstrap Approuch. School of Economics and Management, 1-16.

Battese, G. E., \& Coelli, T. J. (1995). A Model for Technical Inefficiency Effects in a Stochastic Frontier Production Function for Panel Data. Empirical Economics, 20, 325-332.

Booth, R. (2009). For the Student Sports Economics, 42(3).

Carvalho, F. A., Marques, M. C. P., \& Carvalho, J. L. F. (2009). Redes interorganizacionais, poder e dependência no futebol brasileiro. Organizações \& Sociedade, 16(48), 101-121. Retrieved from http://www.spell.org.br/documentos/ver/419/redes- desempenho financeiro no uso de recursos em alguns clubes de futebol, como Palmeiras e Atlético Mineiro.

A contribuição do artigo de ordem técnica está relacionada à aplicação da técnica de regressão e de testes de confiabilidade do modelo proposto, de técnica não paramétrica na geração de scores de eficiência financeira e análise de redes sociais (ARS) na compreensão dos fluxos de jogadores em clubes de futebol que realizaram negociações de jogadores com os clubes semifinalistas do paulista em 2017. Em termos práticos o artigo contribuiu na criação de indicadores de eficiência financeira de clubes de futebol como instrumentos de tomada de decisão gerencial.

Os gestores dos clubes com scores abaixo da fronteira de produção necessitarão repensar suas práticas gerenciais no uso dos recursos de forma a maximizar os resultados organizacionais. Como em toda pesquisa, o estudo apresenta algumas limitações, a contar do tamanho da amostra, do número de variáveis e da dificuldade de coleta de dados nas demonstrações contábeis dos clubes que não são padronizadas. Portanto, se fossem considerados todos os clubes brasileiros do período de análise ou todos os clubes profissionais do mundo do mesmo período, um maior número de variáveis, com dados padronizados, diferentes conclusões seriam alcançadas.

interorganizacionais--poder-e-dependencia-no-futebolbrasileiro

Estender, A. C. (2014). A Importância da administração profissional para os clubes de futebol. Revista Administração Em Diálogo - RAD, 15(3), 18-32.

Frick, B. (2011). Performance, salaries, and contract length: Empirical evidence from German soccer. International Journal of Sport Finance, 6(2), 87-118.

Gerrard, B. (2005). A Resource-Utilization Model of Organizational Efficiency in Professional Sports Teams. Journal of Sport Management, 19, 143-169.

Hair, J. F., Anderson, R. E., Tatham, R. L., \& Black, w. C. (2005). Analise Multivariada de Dados. (J. F. Hair, R. E. Anderson, R. L. Tatham, \& w. C. Black, Eds.) (5th ed.). Porto Alegre: Bookman.

Hanneman, R. A, \& Riddle, M. (2005). Introduction to Social Network Methods. Riverside, CA: University of California, Riverside. On-Line Textbook, 46(7), 5128- 
5130. https://doi.org/10.1016/j.socnet.2006.08.002

Hatala, J. P. (2006). Social Network Analysis in Human Resource Development: A New Methodology. Human Resource Development Review, 5(1), 45-71. https://doi.org/10.1177/1534484305284318

Ji, Y., \& Lee, C. (2009). Data Envelopment Analysis in Stata, (ii), 1-13. under review by the Stata Journal.

Leoncini, M. P., \& Silva, M. T. da. (2005). Entendendo o futebol como um negócio: um estudo exploratório. Gestão \& Produção, 12(1), 11-23. https://doi.org/10.1590/S0104-530X2005000100003

Liu, X. F., Liu, Y. L., Lu, X. H., Wang, Q. X., \& Wang, T. $X$. (2016). The anatomy of the global football player transfer network: Club functionalities versus network properties. PLOS ONE, 11(6), 1-14. https://doi.org/10.1371/journal.pone.0156504

Meza, L. A., Neto, L. B., Mello, J. C. C. B. S. de, Gomes, E. G., \& Coelho, P. H. G. (2005). FREE SOFTWARE FOR DECISION ANALYSIS. A Software Package for Data Envelopment Models, 207-212. https://doi.org/10.5220/0002548802070212

Mourao, P. (2012). The indebtedness of Portuguese soccer teams--looking for determinants. Journal of Sports Sciences, 30(10), 1025-1035. https://doi.org/10.1080/02640414.2012.695085

Mourao, P. R. (2016). Soccer transfers, team efficiency and the sports cycle in the most valued European soccer leagues - have European soccer teams been efficient in trading players? Applied Economics, 48(56), 5513-5524. https://doi.org/10.1080/00036846.2016.1178851
Peeters, T., \& S. Szymanski (2015). Financial Fair Play in European Football. Economic Policy, 29 (78), 343-349. doi:10.1111/ecop.2014.29.issue-78

Penrose, E. T. (1959). The Theory of Growth of the Firm. (E. T. Penrose, Ed.). Blackwell: Oxford. https://doi.org/10.1093/0198289774.003.0002

Pina, T. J., Paulo, A., \& Araújo, D. (2017). Network characteristics of successful performance in association football. A study on the UEFA champions league. Frontiers in Psychology, 8(JUL). https://doi.org/10.3389/fpsyg.2017.01173

Pollard, R., \& Reep, C. (1997). Measuring the Effectiveness of Playing Strategies at Soccer. Journal of the Royal Statistical Society, 46(4), 541-550.

Prearo, L. C., Gouvêa, M. A., \& Romeiro, M. do C. (2012). Avaliação da adequação da aplicação de técnicas multivariadas de dependência em teses e dissertações de algumas instituições de ensino superior. Ensaios FEE, 33(1), 267-296.

Rohde, M., \& Breuer, C. (2016). Europe's Elite Football: Financial Growth, Sporting Success, Transfer Investment, and Private Majority Investors. International Journal of Financial Studies, 4(2), 12. https://doi.org/10.3390/ijfs4020012

Transfermarkt. Mercado de transferências, rumores, valores de Mercado e notícias. Disponível em: http://www.transfermarkt.pt/. Acesso em: 04/03/2018 
Eficiência Financeira, Atores e Interações: Um Estudo do Fluxo de Jogadores entre Clubes e as Equipes Semifinalistas de São Paulo em 2017

\section{SOBRE OS AUTORES}

Edimilson Eduardo da Silva - Universidade Municipal de São Caetano do Sul - USCS, São Caetano do Sul, SP (Brasil). E-mail: edimilsones2013@gmail.com Orcid id: https://orcid.org/0000-0002-6104-7625

Agnaldo Antonio dos Santos - Universidade Municipal de São Caetano do Sul - USCS, São Caetano do Sul, SP (Brasil). E-mail: agnaldoa@outlook.com.br Orcid id: https://orcid.org/0000-0003-3205-9237

Marco Antônio Pinheiro da Silveira - Universidade Municipal de São Caetano do Sul - USCS, São Caetano do Sul, SP (Brasil). E-mail: marco.pinheiro@prof.uscs.edu.br Orcid id: https://orcid.org/0000-0001-6658-5548

Paulo Jorge Reis Mourão - Universidade do Minho, Braga, (Portugal). E-mail: paulom@eeg.uminho.pt Orcid id: https://orcid.org/0000-0001-6046-645X

\section{FINANCIAL EFFICIENCY, ACTORS AND INTERACTIONS: A STUDY OF THE PLAYER FLOW BETWEEN CLUBS AND THE SÃO PAULO SEMIFINALISTS TEAMS IN 2017}

Edimilson Eduardo da Silva, Agnaldo Antonio dos Santos, Marco Antônio Pinheiro da Silveira, Paulo Jorge Reis Mourão Universidade Municipal de São Caetano do Sul - USCS, São Caetano do Sul, (Brasil)

Universidade do Minho, Braga, (Portugal)

\section{ARTICLE DETAILS}

\section{Article history:}

Received: 29 March 2019

Accepted: 29 September 2019

Available online January: 01 th 2020

Double Blind Review System

Scientific Editor

Ilan Avrichir

\section{Key words}

Financial Efficiency

Social Network Analysis

Player Flow

\begin{abstract}
RESUMO
Objective: To discuss the financial efficiency indexes of the Brazilian clubs that stood out in the transfer flows, considering the sample composed by the semifinalist clubs of São Paulo League in 2017.

Method: in the first stage, information about player flows was collected and, In the second stage, data on operating revenue, suppliers, cost of professional athletes and non-professional athletes in clubs in Brazil have also been analyzed.

Main results: Financial efficiency scores provided evidence of the maximum level of resource use by professional and non-professional athletes in generating operating income in Palmeiras and Atletico Mineiro. Regarding the actors, it has been noted that the clubs Grêmio Osasco Audax and Cruzeiro Esporte Clube made more players available for the semifinalist clubs. In the player flows between clubs from Brazil and other countries, the teams from Italy and Portugal stood out. Relevance/ Originality: This work identified clubs' maximum resource efficiency scores in generating operating income, key players, and interactions in player streams.

Methodological contributions: Simultaneous use of parametric and nonparametric techniques such as Multiple Linear Regression, Data Envelopment Analysis (DEA) and Social Network Analysis (ARS).

Contributions to management: creation of financial efficiency indicators as tools to support management decisions in football clubs.
\end{abstract}




\section{EFICIENCIA FINANCIERA, ACTORES E INTERACCIONES: UN ESTUDIO DEL FLUJO DE JUGADORES ENTRE CLUBS Y LOS EQUIPOS SEMIFINALISTAS DE SAO PAULO EN 2017}

Edimilson Eduardo da Silva, Agnaldo Antonio dos Santos, Marco Antônio Pinheiro da Silveira, Paulo Jorge Reis Mourão Universidade Municipal de São Caetano do Sul - USCS, São Caetano do Sul, (Brasil)

Universidade do Minho, Braga, (Portugal)

\section{DETALLES DEL ARTÍCULO}

Historia del Artículo:

Recibido: 29 Marcha 2019

Aceptado: 08 Septiembre 2019

Disponible en línea: 01 de enero 2020

\section{Double Blind Review System}

\section{Editor Científico}

Ilan Avrichir

\section{Palabras-clave:}

Eficiencia Financiera

Análisis de Redes Sociales

Flujo de Jugadores

\begin{abstract}
RESUMO
Objetivo: presentar los índices de eficiencia financiera de los clubs brasileños, los actores y las interacciones que se destacaron en los flujos de jugadores con los clubs semifinalistas de São Paulo en 2017.

Método: en la primera etapa, se extrajeron informaciones sobre el flujo de jugadores y, en la segunda, datos sobre la receta operacional, proveedores, coste de atletas profesionalizados y no profesionalizados en la generación de receta operacional en Palmeiras y Atlético Mineiro. Referente a los actores, los clubs Gremio Osasco Audax y Cruzeiro Esporte Club disponibilizaron más jugadores para los clubs semifinalistas. En los flujos de jugadores entre clubs de Brasil y de otros países, se destacaron los equipos de Itália y de Portugal.

Relevancia/ originalidad: demostrar os scores de eficiencia máximo de los clubs en el uso de recursos en la generación de receta operacional, los actores de relieve y las interacciones en los flujos de jugadores.

Contribuciones metodológicas: uso simultáneo de técnicas paramétricas y no paramétricas como la Regressión Linel Múltiple, el Análisis Envoltorio de Datos (DEA) y el Análisis de Redes sociales (ARS).

Contribuciones para la gestión: creación de indicadores de eficiencia financiera como herramientas de apoyo a la toma de decisiones gerenciales en clubs de fútbol.
\end{abstract}

\section{Para citar este artigo:}

Silva, E., Santos, A., Silveira, M., \& Mourão, P. (2020). Eficiência Financeira, Atores e Interações: Um Estudo do Fluxo de Jogadores entre Clubes e as Equipes Semifinalistas de São Paulo em 2017. Internext, 15(1), 88-103. doi: http://dx.doi.org/10.18568/internext.v15i1.538 